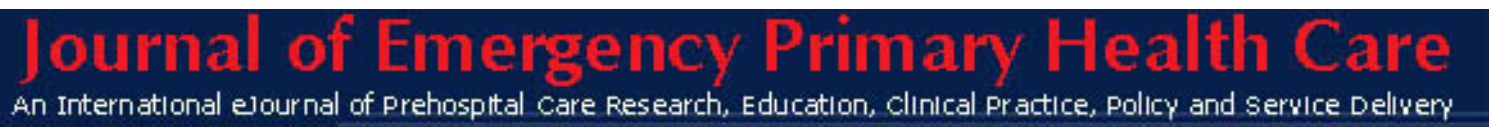

ISSN 1447-4999

\title{
EVIDENCE BASED PRACTICE
}

Article 990202

\section{Introduction of advanced care to prehospital services in Québec Executive Summary - May 2005}

Prepared by

Dr. Reiner Banken MD, MSc (Community Health), Consultant Researcher, AETMIS

Brigitte Côté MD, MSc (Public Health), Consultant Researcher, AETMIS

François de Champlain MD, FRCPC, F.AC.E.P Emergency Medicine Specialist André Lavoie PhD (Epidemiology), Epidemiologist

This report was translated from an official French publication of the Agence d'évaluation des technologies et des modes d'intervention en santé (AETMIS). Both the original report titled Introduction des soins médicaux avancés dans les services préhospitaliers d'urgence au Québec and the English report are available in PDF format on the Agency's Web site.

\section{Scientific review}

Dr. Véronique Déry, MD, MSc, Chief Executive Officer and Scientific Director Jean-Marie R. Lance, MSc, Senior Scientific Advisor

\section{Translation}

Jocelyne Lauzière, MA, Certified Translator

\section{Editorial supervision}

Suzie Toutant

\section{Proofreading}

Frédérique Stephan

\section{Page layout}

Frédérique Stephan

\section{Bibliography research}

Micheline Paquin

Co-ordination

Lise-Ann Davignon 


\section{Communications and dissemination}

Richard Lavoie, MA

For further information about this publication or any other AETMIS activity, please contact:

Agence d'évaluation des technologies et des modes d'intervention en santé

2021 Union Avenue, Suite 1050

Montréal (Québec) H3A 2S9

Telephone: (514) 873-2563

Fax: (514) 873-1369

E-mail: aetmis@aetmis.gouv.qc.ca

www.aetmis.gouv.qc.ca

\section{How to cite this document:}

Agence d'évaluation des technologies et des modes d'intervention en santé (AETMIS). Introduction of advanced life support in pre-hospital emergency medical services in Québec. Report prepared by Reiner Banken, Brigitte Côté, François de Champlain and André Lavoie (AETMIS 05-01). Montréal: AETMIS, 2005.

\section{Legal deposit}

Bibliothèque nationale du Québec, 2005

Library and Archives Canada, 2005

ISBN 2-550-44471-X (Print) (French edition ISBN 2-550-44197-4)

ISBN 2-550-44472-8 (PDF) (French edition ISBN 2-550-44484-1)

(C) Gouvernement du Québec, 2005.

This report may be reproduced in whole or in part provided that the source is cited.

\section{MISSION}

The mission of the Agence d'évaluation des technologies et des modes d'intervention en santé (AETMIS) is to contribute to improving the Québec health-care system and to participate in the implementation of the Québec government's scientific policy. To accomplish this, the Agency advises and supports the Minister of Health and Social Services as well as the decision-makers in the health care system, in matters concerning the assessment of health services and technologies. The Agency makes recommendations based on scientific reports assessing the introduction, diffusion and use of health technologies, including technical aids for disabled persons, as well as the modes of providing and organizing services. The assessments take into account many factors, such as efficacy, safety and efficiency, as well as ethical, social, organizational and economic implications. 


\section{EXECUTIVE}

Dr. Luc Deschênes

Cancer Surgeon, President and Chief Executive Officer of AETMIS, Montréal, and

Chairman,

Conseil médical du Québec, Québec

Dr. Véronique Déry

Public Health Physician, Chief Executive

Officer and Scientific Director

Dr. Alicia Framarin

Physician, Deputy Scientific Director

Jean-Marie R. Lance

Economist, Senior Scientific Advisor

\section{BOARD OF DIRECTORS}

\section{Dr. Jeffrey Barkun}

Associate Professor, Department of Surgery,

Faculty of Medicine, McGill University, and Surgeon, Royal Victoria Hospital (MUHC), Montréal

\section{Dr. Marie-Dominique Beaulieu}

Family Physician, Holder of the Dr. Sadok Besrour Chair in Family Medicine, CHUM, and Researcher, Unité de recherche évaluative, Hôpital Notre-Dame (CHUM), Montréal

\section{Dr. Suzanne Claveau}

Specialist in microbiology and infectious diseases, Hôtel-Dieu de Québec (CHUQ), Québec

\section{Roger Jacob}

Biomedical Engineer, Coordinator, Capital Assets and Medical Equipment, Agence de développement de réseaux locaux de services de santé et de services sociaux de Montréal, Montréal

\section{Denise Leclerc}

Pharmacist, Board Member of the Institut universitaire de gériatrie de Montréal, Montréal

\section{Louise Montreuil}

Assistant Executive Director, Direction générale de la coordination ministérielle des relations avec le réseau, ministère de la Santé et des Services sociaux, Québec

\section{Dr. Jean-Marie Moutquin}

Obstetrician/Gynecologist, Research Director, and Executive Director, Département d’obstétrique-gynécologie, CHUS, Sherbrooke 


\section{Dr. Réginald Nadeau}

Cardiologist, Hôpital du Sacré-Cœur, Montréal, Board Member of the Conseil du médicament du Québec

\section{Guy Rocher}

Sociologist, Professor, Département de sociologie, and Researcher, Centre de recherche en droit public, Université de Montréal, Montréal

\section{Lee Soderström}

Economist, Professor, Department of Economics, McGill University, Montréal

\section{FOREWARD}

In the fall of 2004, several Québec emergency medical technicians obtained Ontario certification in advanced paramedic care and asked for authorization to apply their training here. It was in this context that the Minister of Health and Social Services asked AETMIS (Agence d'évaluation des technologies et des modes d'intervention en santé) in November 2004 to prepare a report on the role of advanced care, also known as advanced life support (ALS), in the organization of pre-hospital emergency services in Québec.

The present assessment is based primarily on a comprehensive review of the scientific literature on this topic. The analysis also took into account developments in pre-hospital care, advanced-care practices and training, internationally as well as in Canada and in Québec.

Examination of scientific data led to four major findings. First, there is not enough solid evidence to warrant the generalized introduction of advanced pre-hospital care throughout Québec. Second, some preliminary evidence shows that advanced care could be beneficial, especially in the case of respiratory distress or cardiac chest pain. Third, the limited amount of available evidence indicates that advanced care is neither beneficial nor detrimental in terms of mortality or morbidity in patients experiencing non-traumatic cardiopulmonary arrest, although the hypothesis that this type of care may be beneficial remains plausible. Finally, evidence reveals that advanced care is associated with adverse effects in certain circumstances, such as the endotracheal intubation of young children and the treatment of trauma.

In light of these findings, current developments and Québec's particular context, AETMIS recommends that, for the time being, Québec limit the use of advanced care to duly evaluated pilot projects, with priority being given to the treatment of respiratory distress, chest pain and cardiopulmonary arrest. AETMIS also recommends implementing a series of measures designed to optimize both primary pre-hospital care and the chain of survival throughout Québec, chiefly by enhancing the training provided to emergency medical technicians.

In submitting this report, AETMIS hopes to inform the ongoing debate on the introduction of advanced care to Québec's pre-hospital services.

\section{Dr. Luc Deschênes}

President and Chief Executive Officer 


\section{ACKNOWLEDGEMENTS}

This report was prepared at the request of the Agence d'évaluation des technologies et des modes d'intervention en santé (AETMIS) by Reiner Banken, MD, MSc (Community Health), Consultant Researcher, AETMIS, Brigitte Côté, MD, MSc (Public Health), Consultant Researcher, AETMIS, François de Champlain, MD, FRCPC, F.AC.E.P Emergency Medicine Specialist, and André Lavoie, PhD (Epidemiology), Epidemiologist.

We would like to thank Stephane Perron, MD, MSc (Public Health), for his contribution to the quality control of several sections of the present report.

AETMIS would like to thank the external reviewers of this report for their valuable feedback:

\section{Marica Ferri, MD}

Co-ordinator, Unità di Progetto: EBM e Modelli Assistenziali, Agenzi di Sanità Pubblica della Regione Lazio, Rome, Italy

\section{Assunta De Luca, MD}

Head of Emergency Unit, Unità di Progetto: EBM e Modelli Assistenziali, Agenzi di Sanità Pubblica della Regione Lazio, Rome, Italy

\section{Alphonse Montminy, MD}

President, Comité des soins d'urgence cardiaque, Fondation des maladies du cœur du Québec, Montréal, Québec

\section{Joseph J. Osterwalder, MD}

Head of Emergency Department, Kantonsspital St. Gallen, Switzerland

\section{Pierre Poirier}

Executive Director, Paramedic Association of Canada, and Chairman, National Occupational Competency Profiles for Paramedic Practitioners Review Committee, Ottawa, Ontario

\section{Wayne Smith, MD}

Medical Director, Services préhospitaliers d'urgence, Agence de développement de réseaux locaux de services de santé et de services sociaux de l'Estrie, Sherbrooke, Québec

The following individuals greatly contributed to this report by providing information, support and key advice:

\section{Marcel Boucher, MD}

Director, Services professionnels et de l'assurance de la qualité, Corporation d'urgences-santé, Montréal, Québec 


\section{Robert Burgess, ACP, AEMCA}

Senior Manager, Division of Prehospital Care, Sunnybrook-Osler Centre for Prehospital Care, Toronto, Ontario

\section{Mario Deschênes, MD}

Assistant Syndic, Collège des médecins du Québec, Montréal, Québec

\section{Claude Desrosiers}

Co-ordinator, Module de l'assurance de la qualité et de la formation clinique, Corporation d'urgences-santé, Montréal, Québec

\section{Daniel Lefrançois, MD}

Medical Director, Direction adjointe des services préhospitaliers d'urgence, ministère de la Santé et des Services sociaux, Quebec City, Québec

\section{Suzanne Michalk, MD}

President, Comité sur les soins préhospitaliers d'urgence, Collège des médecins du Québec, Montréal, Québec

\section{Gisèle Ouimet}

Research Consultant, Module de l'assurance de la qualité et de la formation clinique, Corporation d'urgences-santé, Montréal, Québec

\section{Eli Segal, MD}

Research Co-ordinator, Module de recherche, Corporation d'urgences-santé, Montréal, Québec

\section{Ian Stiell, MD}

Senior Scientist, Clinical Epidemiology Clinic, Loeb Health Research Institute, and principal investigator, OPALS study, Ottawa, Ontario

\section{Brian Schwartz, MD}

Medical Director, Division of Prehospital Care, Sunnybrook-Osler Centre for Prehospital Care, Toronto, Ontario, and President, Canadian Relations Ad Hoc Committee, National Association of EMS Physicians (USA)

\section{DISCLOSURE OF CONFLICTS OF INTEREST}

Dr. François de Champlain is also a physician with the Corporation d'urgences-santé. André Lavoie was in charge of research and quality assurance for the Corporation d'urgences-santé from 1992 to 1999. He worked as a consultant for JSS Medical Research, especially in the context of a binding contract between this firm and the Corporation d'urgences-santé. Dr. de Champlain and André Lavoie informed AETMIS of this situation well before this work began. 


\section{SUMMARY INTRODUCTION}

In November 2004, the Minister of Health and Social Services asked AETMIS (Agence d'évaluation des technologies et des modes d'intervention en santé) to prepare a report on the role of advanced life support in the organization of pre-hospital emergency medi-cal services in Québec. Based on an analysis of the scientific evidence of the efficacy and safety of this type of care, and of the conditions required to achieve effec-tiveness and safety objectives in the Québec context, this report formulates a series of recommendations designed to provide the scientific basis for the policy directions to be taken by the Ministère de la Santé et des Services sociaux in this regard.

\section{NATURE AND STRUCTURE OF PRE-HOSPITAL EMERGENCY CARE}

Primary care, also known as basic life support (BLS), currently consists of a spectrum of non-invasive procedures, that is, those limited to external application such as using direct pressure and bandages to control hemorrhaging, immobilizing suspected fractures, maintaining respiratory function, maintaining circulatory function by means of chest compressions (cardiopulmonary resuscitation) and, for the past few years, administering five types of medication in accordance with very strict protocols. Some of these emergency procedures can be performed by bystanders or by first responders. All primary-care procedures can be performed by emergency medical technicians (EMT). Advanced care, or advanced life support (ALS), implies invasive procedures, such as endotracheal intubation, intravenous access (inserting a catheter into a vein), administering different types of medication, and fluid resuscitation (replacing blood loss by administering fluids in order to increase blood pressure and improve oxygenation of body tissues and organs).

The line between primary care and advanced care based on the invasiveness of the procedures used is becoming increasingly arbitrary, owing to the introduction of new procedures and the shift of certain advanced-care practices to primary care. This division is now based more on the training given to advanced-care practitioners, who must have greater background knowledge in health sciences and physical sciences to be able to use more complex care protocols safely and effectively.

In Canada, the regulation of pre-hospital practice is a provincial jurisdiction, and each legislature is free to determine its own practitioner classification and to set practice conditions. However, training standards for practitioners of pre-hospital emergency care were standardized in 2001 with the development of the National Occupational Competency Profiles for Paramedic Practitioners. These profiles consist of four different levels: emergency medical responder, primary care paramedic, advanced care paramedic and critical care paramedic. Most Canadian provinces are currently shifting toward this model.

A distinction must be made between level of training and level of practice in the provinces and regions. For example, Québec seems to be the only Canadian province where the Combitube ${ }^{\circledR}$ (an orotracheal tube with two ventilation ports) is used in the context of primary care. Elsewhere in North America, this technique is used mainly in 
advanced care when endotracheal intubation has failed. Inserting intravenous lines (one of the core characteristics of advanced care) is making its way into primary care in Alberta and in certain regions of Ontario, although this procedure is performed only to administer dextrose to hypoglycemic patients.

\section{HISTORY OF PRE-HOSPITAL EMERGENCY MEDICAL SERVICES IN QUÉBEC}

In Québec, the history of ambulance services was long marked by local and even individual initiatives. In the 1950s and 1960s, ambulance services were often run by funeral parlours. By the late 1970s, ambulance permits were being issued by the Ministère de la Santé et des Services sociaux (MSSS), which later delegated this responsibility to the regional health and social services boards. In the Montreal region, in the 1980s and 1990s, various changes to the pre-hospital system led to the creation of Urgences-santé, which until 2002 employed physicians to provide advanced care. Else $\neg$ where in Québec, the different regions were developing systems based exclusively on primary care dispensed by emergency medical technicians.

Since the 1990s, the history of pre-hospital care in Québec has been marked by two major development-planning initiatives. In 1992, the report Chaque minute compte (Every minute counts) was the first to propose that the various structures making up Québec's pre-hospital emergency system be organized into a consistent whole. It did not include any position statement on the usefulness of advanced care. Published in 2000, the Dicaire report laid out directions for developing Québec's pre-hospital emergency system, focusing mainly on the relevance of extending the chain of survival throughout the province. This report also argued for conducting field evaluations of advanced-care procedures in structured pilot projects rather than broadening the use of the full spectrum of procedures.

The Dicaire report also paved the way for the adoption in 2002 of the Act respecting prehospital emergency services, which now frames the organization of these services provincewide. In addition, the use of advanced-care protocols is now permitted by virtue of the Regulation respecting the professional activities that may be engaged in within the framework of pre-hospital emergency services, adopted under Québec's Professional Code.

Over the past few years, Québec has made a sustained effort to extend the chain of survival throughout its jurisdiction in a bid to limit regional disparities insofar as possible. While other Canadian provinces have chosen the route of developing both primary care and advanced care, Québec has made rapid strides in expanding both the scope and the geographic range of primary care.

\section{RESULTS}

The effectiveness and safety of advanced life support were analyzed through a comprehensive review of relevant scientific literature. Special attention was given to the evaluation of a pre-hospital advanced life support program in Ontario: the OPALS 
(Ontario Prehospital Advanced Life Support) study. This is probably the largest study ever undertaken on advanced life support, in terms of both the number of patients included in the study and the type of study design used, a before-after non-randomized comparative trial. Data on over 30000 patients were compiled over a ten-year period (1994-2004).

\section{Cardiopulmonary arrest}

In the case of cardiac arrest, many studies have clearly established the efficacy of the first links in the chain of survival: early call to 9-1-1, early cardiopulmonary resuscitation (cardiac massage and ventilation) and early defibrillation.

In a system such as the one in Ontario, where early defibrillation is performed by paramedics, the addition of an ALS medication protocol does not improve patient survival. The OPALS study concludes that it is important for the general public to be trained in cardiopulmonary resuscitation (CPR) and for first responders to be able to perform early defibrillation.

\section{Other cardiac problems}

In the case of myocardial infarction, many publications have shown that early access to thrombolysis or angioplasty reduces mortality. If a patient with suspected cardiac chest pain is to be transported directly to a tertiary-care centre, a 12-lead electrocardiogram (ECG) must be performed in the pre-hospital setting. Studies with varying levels of methodological rigour have examined this question and shown an increase in the use of thrombolysis or primary angioplasty, along with a significant reduction in treatment delays when ECGs are obtained in the field. Similarly, certain studies have revealed that ECG results can be transmitted to the receiving hospital so that the interpretation can be confirmed.

In the OPALS study, treatment of suspected cardiac chest pain in accordance with ALS medication protocols led to a significant reduction in mortality rates, which dropped from $4.3 \%$ to $3.2 \%$, and more specifically, from $13.1 \%$ to $8.5 \%$ for myocardial infarc-tion. Abstracts of this study have been published and the results have been pre-sented at several conferences. Publication of the full OPALS study should shed greater light on the conditions liable to explain the effects reported. It is currently impossible to know the extent to which this increased survival rate after cardiac chest pain is attributable to the two most com-monly prescribed medications, aspirin and nitro-glycerine, which are already included in the symptom-relief program of five drugs that is part of Québec's prehospital BLS care. Note that medication protocols are different in basic life support and advanced life support. Advanced care paramedics receive more in-depth training, which allows them to follow protocols requiring more acute clinical judgment to be able to administer the drugs safely.

\section{Respiratory problems}

Respiratory problems represent a significant proportion of the use of pre-hospital emergency services. According to the data provided by Urgences-santé, 11.1\% of all the patients it transported in 2003 had respiratory problems, as assessed by ambu-lance 
technicians, representing a total of 16366 patients. In Ontario, in-hospital mor-tality of patients in respiratory distress transported by ambulance was $18 \%$. In hospital emergency departments (EDs), the care of patients with congestive heart failure has been greatly improved through the use of non-invasive positive pressure ventilation, such as CPAP (continuous positive air-way pressure). Certain studies have shown the benefits of CPAP in pre-hospital settings, but it seems prudent to wait for the published results of an inprogress random-ized clinical trial on the usefulness of this approach.

In the OPALS study, the treatment of respiratory distress in compliance with ALS medication protocols has led to a significant drop in mortality rates, from $14.3 \%$ to $12.3 \%$. If we examine the different diagnosis subgroups, however, a statistically significant reduction in mortality (from $15.1 \%$ to $11.0 \%$ ) was documented only in patients with congestive heart failure. The improved survival rates in these patients are indeed impressive; however, as is the case with cardiac chest pain, we have only abstracts and conference presentations to rely on. Once published, the complete OPALS study will probably explain the effects reported. Unlike the medications used for cardiac chest pain, those for congestive heart failure are not included in the symptom-relief program that is part of Québec’s pre-hospital basic life support care.

\section{Trauma}

For the victims of major trauma, the interval between the arrival of emergency personnel and access to definitive trauma care is critical-approximately $80 \%$ of deaths occur within the first minutes or hours following the trauma. The tertiary trauma centres established in Québec have helped reduce intrahospital delays and mortality rates. Over the past ten years or so, all published studies and systematic reviews have indicated that the transportation of trauma patients should not be delayed by advanced life support.

\section{Pediatrics and advanced care}

Few pre-hospital studies have addressed the effectiveness of pediatric advanced life sup-port (PALS), mostly because of the rela-tively small number of children needing emergency care. Endotracheal intubation is one exception. A randomized controlled trial reveals that this procedure is difficult, if not dangerous, if performed as part of pediatric advanced life support. Since the release of that study, several pre-hospital emergency medical services (EMS) systems have halted this practice with children.

\section{Other avenues}

For other conditions, such as seizures, pain, severe hypoglycemia, strokes and opioid overdoses, advanced life support could be beneficial, but evidence is either lacking or not conclusive enough to justify introducing care protocols.

\section{FIELD EVALUATION OF ADVANCED LIFE SUPPORT AT URGENCES- SANTE}

The infrastructure built by Urgences-santé is more highly developed than that found elsewhere in Québec. This organization has a detailed information system, medical control through highly experienced pre-hospital emergency physicians, a well-established 
quality-assurance program and a training centre. It has been developing a pre-hospital research program for the past twenty years. Consequently, when the Dicaire report was published in 2000, Urgences-santé already had an environment that was favourable to conducting field evaluations of ALS protocols by a cohort of emergency medical technicians whose training was tailor-made for this project. Five advanced life support protocols were scheduled to be tested: 1) endotracheal intubation; 2) use of Magill forceps to remove foreign bodies from airways; 3) administration of vasopressin and amio-darone in the case of non-refractory (shockable) cardiac arrhythmia; 4) admini-stration of epinephrine and atropine in the case of refractory (shock-resistant) cardiac arrhythmia; and 5) intravenous administration of dextrose in the case of severe hypoglycemia. The evaluation project was based on a quasi-experimental design.

Because of a series of modifications to the advanced life support project, the exposure of the eighteen emergency medical techni-cians trained in ALS was much more limited than originally anticipated. As a result, the original research study design had to be abandoned. It was nevertheless possible to evaluate the effectiveness of two of the five protocols related to cardiac arrest. Results were comparable to those observed by the OPALS researchers in Ontario and matched other outcomes documented in the literature: for cardiac arrest, ALS care seemed to provide very short-term results (e.g., increased return of spontaneous circulation or survival to hospital admission) but did not lead to any improvements in rates of survival to hospital discharge, compared with basic life support care.

\section{EVALUATION LIMITATIONS}

Our assessment rests on an evidence-based model that includes both scientific evidence and contextual information. These data, however, are limited in several respects. First, scientific evidence in this field is scarce and its validity is weakened by the use of study designs that lack rigour. Published results deal more often with mortality, whereas effects on morbidity, quality of life or patient satisfaction deserve further study since they are liable to affect decisions regarding the organization of pre-hospital emergency medical services. Furthermore, highly promising results, especially those from the OPALS study, are available only in the form of abstracts or conference presentations. Second, there is also very little contextual information available because most of the regions in Québec have sub-optimal information systems. Given the lack of economic evidence, it also was impossible to produce analyses establishing the cost-effectiveness of different scenarios for developing pre-hospital emergency medical services in Québec.

\section{CONCLUSIONS AND RECOMMENDATIONS}

In his request, the Minister wanted the report prepared by AETMIS to cover four issues:

- the safety of the advanced-care proce $\neg$ dures applied in North American prehospital emergency systems; 
- the effectiveness and efficiency of the care practices that these systems offer to their target populations;

- the role of performance in the chain of survival as a factor independent of the medical procedures performed by advanced-care technicians or paramedics; and

- the identification of the optimal path to take for developing pre-hospital services in Québec, taking into account the importance to be given to each of the links in the chain of survival within the context of the system's overall efficiency with respect to the whole population.

This assessment is based on a review of relevant scientific literature and an analysis of developments in pre-hospital care, internationally, in Canada and in Québec, including their advanced-care training programs and practices.

Examination of the scientific evidence on the efficacy and safety of advanced prehospital care led to four major findings:

- There is currently not enough solid evidence to support the generalized introduction of an advanced-care program throughout Québec.

- Preliminary data show that advanced care could be beneficial, especially in the case of respiratory distress and cardiac chest pain.

- The limited evidence that is available indicates that advanced care is neither beneficial nor detrimental in terms of mortality or morbidity in patients experi-encing non-traumatic cardiopulmonary arrest, yet the hypothesis that it might be beneficial in reducing morbidity and mortality has not yet been set aside and deserves further research.

- Data indicate that advanced care is associated with adverse effects in certain circumstances, mainly the endotracheal intubation of young children and the treatment of trauma cases in general.

This evidence, combined with field data and contextual information, leads us to recommend that Québec should follow a reasoned approach to introducing advanced prehospital care. This approach should be based on the vision of making optimal use of both the full range of pre-hospital care and the various links in the chain of survival. It is therefore recommended that specific measures be taken to expand advanced emergency care and to optimize primary pre-hospital care, including cardiopulmonary resuscitation and cardiac defibrillation. It would also be advisable to implement measures of a more general nature that affect both primary and advanced pre-hospital care.

\section{Reasoned approach to introducing pre-hospital advanced life support}

The adoption of a reasoned approach to introducing advanced pre-hospital care will respond to two issues linked to its implementation in Québec. The first issue concerns giving consideration to the scientific evidence and information gathered during this 
analysis. Our assessment shows that advanced pre-hospital care is a field particularly devoid of high-quality research. Yet, in spite of this lack of evidence, it must be acknowledged that simply because the efficacy of these procedures has not been demonstrated, it does not mean that they are ineffective. Furthermore, our talks with international, Canadian and Québec experts revealed that our reflection on this matter must include the lessons drawn over the years from field experience in advanced emergency care, along with anticipated developments in this area. The second issue concerns translating all these data into coherent avenues to action tailored to the specific conditions in Québec as a whole and in its different regions. By reasoned implementation approach, we mean a gradual, reflective process designed to make optimal use of resources within the context of an innovation: we must understand its mode of operation in context, measure its effects, and draw conclusions about its portability or applicability to other practice situations.

Recommendation 1: It is recommended that the use of advanced-care procedures in Québec be limited, for the time being and as an initial step, to pilot field projects.

Rationale: The decision to immediately introduce the full range of advanced-care procedures as defined in the National Occupational Competency Profiles for Paramedic Practitioners could be fuelled by considerations such as workforce mobility and standardization, or yet again by the possibility and usefulness of taking part in multicentre research studies on the efficacy of these standards. That decision, however, is not at this moment supported by evidence. The potential for severe adverse effects tied to advanced pre-hospital care in trauma cases and to the endotracheal intubation of children calls for caution. Data shedding light on these harmful effects were not available thirty years ago when these procedures were introduced into practice in other parts of the world. On the other hand, we cannot keep silent about the significant lifesaving potential of pre-hospital care for patients with respiratory distress or cardiac chest pain. The Dicaire report understandably recommended not to make the full panoply of advanced care available in Québec's pre-hospital system, but to run trials within structured, timelimited pilot projects armed with an evaluation mechanism that would show that this type of care could offer clinical added value in terms of mortality and morbidity. The importance of the links between different contextual factors and the efficacy and safety of advanced care calls for the establishment of pilot projects that will inform the effectiveness of this care in different settings in Québec. In fact, it would be important to launch these pilot projects as soon as possible so that the eighteen emergency medical technicians trained in advanced care can maintain and make full use of the skills and competencies they have acquired and so that the entire Québec population can quickly benefit from the interventions that will have been proven to be effective.

Recommendation 2: It is recommended that pilot projects be set up in Québec with a view to assessing the effectiveness and efficiency of advanced-care protocols and to evaluating the organizational conditions required for their implementation, with priority being given to respiratory distress, chest pain and cardiopulmonary arrest. These projects, which may be carried out in any region of the province, must nevertheless meet the following conditions: 
a) They must be carried out in a pre-hospital emergency service capable of guaranteeing to the MSSS that it will comply with high standards with respect to both staff training and quality control of the procedures.

b) The pre-hospital service must offer rigorous medical control, whether on-line or onsite, provided by physicians with expertise in emergency medicine and pre-hospital care.

c) The evaluation must be conducted under the scientific direction of a research group recognized for its independence and experience.

d) Given that evaluation of the effects of advanced care will contribute to the advancement or application of knowledge in this field, it must be based on an experimental or a quasi-experimental study design approved by a research-granting agency or other recognized body.

e)The nature and scope of the project, the minimum standards of medical control to be observed and the reasons that would justify terminating the pilot project prematurely shall be jointly determined by the MSSS, the Collège des médecins du Québec, pre-hospital emergency service authorities, and the researchers concerned.

f) The number of emergency medical technicians well trained in advanced care shall be increased to obtain as quickly as possible enough exposure to guarantee the validity of the evaluation results.

g) Advanced-care protocols introduced shall explicitly exclude children and trauma patients for the time being.

h) The implementation of advanced-care protocols must be evaluated on an ongoing basis so that appropriate adjustments may be identified and put into effect.

Rationale: The Dicaire report recommended that certain advanced-care practices be implemented in certain regions of Québec, provided that the regions were able to comply with implementation requirements. It also suggested that the introduction of advancedcare techniques should be subjected to objective scientific evaluation over a reasonable period of time. The field evaluation of certain advanced-care protocols at Urgences-santé did not apply to patients with chest pain or respiratory distress, where the potential to save lives currently seems to be greatest. Despite the mitigated results of advanced-care procedures in the case of cardiopulmonary arrest, several factors urge us to view them as priorities in these pilot projects. We believe there is a need to define more valid outcome measures of the efficacy of advanced care and to identify the ethical and organizational issues raised by the possibility of performing advanced cardiac-resuscitation procedures in a pre-hospital setting rather than in a hospital emergency department, clearly on condition that the quality of care would be the same as in a hospital. Pilot-project evaluation requires not only that resources be earmarked for research in this area but also that a sufficient number of emergency medical technicians receive training in advanced 
care, in addition to the eighteen who have already completed their training, so as to give appropriate power to conduct a statistical analysis of the outcomes. Other key conditions for the success of this evaluative research include dynamic medical control provided by physicians with expertise in emergency care, and the availability of effective information systems. Urgences-santé seems in many respects to be the ideal place to test these new advanced-care techniques. Nevertheless, given the importance of organizational context in the provision of pre-hospital care in each of Québec's regions, it will be necessary to sustain research efforts in both urban and rural settings.

Recommendation 3: It is recommended that a research program be established that deals specifically with evaluating advanced pre-hospital care and that calls upon the entire research community of Québec, under the leadership of the MSSS.

Rationale: It would be advisable to officially recognize this research area and to allocate budgets for it, if we want to obtain evidence that will be useful for Québec and that will contribute to enhancing the knowledge base in this field at an international level. A process for approving advanced-care protocols for treating suspected cardiac chest pain and respiratory distress that is recognized across Canada and by the Collège des médecins should be initiated in parallel with the implementation of the research program. The research program itself should be methodologically rigorous and should take care to include research activities that meet regional needs. The development of a process for formalizing the evaluation criteria for these pilot projects, and an appeal to the entire scien $\neg$ tific community of Québec, would appear to be two prerequisites for maximizing results.

Recommendation 4: It is recommended that, to ensure throughout Québec a gradual introduction of proven advanced pre-hospital services that keep pace with evolving knowledge, a service-development plan should be established that provides for the training of a sufficient number of emergency medical technicians capable of administering this care, and that sets out appropriate organizational conditions that would include building close partnerships between pre-hospital and hospital settings.

Rationale: It is expected that over the next few years new knowledge will emerge from the studies published by OPALS and other research groups. This knowledge should allow the clear identification of proven advanced-care practices. Moreover, since the release of the Dicaire report, we now have the National Occupational Competency Profiles (NOCP) that provide benchmarks for paramedics working in Canada. Note, however, that increasing the number of emergency medical technicians trained in advanced care will be restricted by the limited availability of teaching staff and material resources that are essential for this training. With a view to developing and maintaining competencies and fostering mutually beneficial collaboration, it would be desirable for Québec to follow the example of other Canadian provinces and build close working partnerships between pre-hospital and hospital settings. In that sense, we could conceivably establish a model along the lines of Ontario's base hospitals, especially in Québec's regions, which generate a lower volume of emergency calls than does the greater Montreal region. This 
would also allow certain regions to have direct on-line support provided by a basehospital emergency physician. Finally, emergency medical technicians must be able to practise within organizational structures that support the delivery of high-quality services. These structures will need to be created or further developed.

\section{Optimizing primary pre-hospital care and the chain of survival}

Recommendation 5: It is recommended that new procedures be included within primary pre-hospital services, provided that this decision is backed by evidence or by experts' recognition that these new procedures have a significant potential for reducing mortality and morbidity.

Rationale: The development of Québec's pre-hospital emergency system was spearheaded by an expert committee that had worked without the benefit of a structured analysis of scientific data. Even though there is often no scientific evidence for certain procedures, they may still have significant potential for reducing mortality and morbidity: this is the case of the Combitube, which is now a standard part of primary pre-hospital care in Québec. This is equally true for the five medications in the Symptom Relief Program, which was implemented both in Québec and in other provinces even before its effectiveness had been proven. Continuous quality improvement of pre-hospital care requires a reasoned approach to adapting the services to new knowledge.

Recommendation 6: It is recommended that the basic training provided to emergency medical technicians be enhanced so that competencies acquired through this training match those stipulated in the National Occupational Competency Profiles (NOCP) for primary care paramedics.

Rationale: Several procedures that were once considered part of advanced care now belong to primary care, and today's advanced pre-hospital practices may very well become the primary practices of tomorrow. A recent example of this trend is the fact that emergency medical technicians are now authorized to administer five medications to patients for symptom relief, a procedure that used to be exclusively part of advanced care. Several factors argue in favour of enhancing the training of emergency medical technicians: the critical nature of situations requiring pre-hospital care; the complexity of the medical procedures performed; the technical support needed by advanced-care emergency medical technicians; and their need to rely on both general and specific skills and competencies in the field. Experience shows that training levels must also be upgraded so that emergency medical technicians can master the new procedures that will be added to current primary care. Furthermore, this extra training would allow them to take part in research projects on primary care.

Recommendation 7: It is recommended that measures be implemented to expand the general public's training in cardiopulmonary resuscitation (CPR) and to ensure that patients experiencing cardiac arrest have access to early defibrillation performed by first responders or bystanders. 
Rationale: Basic cardiopulmonary resuscitation and early defibrillation performed by bystanders (public access defibrillation programs), first responders or emergency medical technicians significantly improve the survival rate of cardiac arrest victims. In the Ontario system, these procedures have effectively been linked to improved survival in patients.

\section{Ensuring other conditions required to optimize the full spectrum of pre-hospital emergency care in Québec}

Recommendation 8: It is recommended that the following be introduced throughout Québec: an enhanced continuing education program; effective medical control; qualityassurance tools; and information systems, such as electronic databases to keep a record of the patients served, their particular health problems, the clinical procedures performed by pre-hospital personnel, and the immediate effects of these procedures.

Rationale: The concept of medical control of pre-hospital care seems present in all countries, but in practical terms it can take the form of direct (on-line) medical support, delegated acts stipulated in standard protocols, or indirect medical support (offline). Supervision to ensure the quality of the procedures is also a key element. Certain provincial regulations governing pre-hospital care dictate that quality-assurance mechanisms be set in place. These mechanisms can include mandatory continuing education, mandatory re-certification, audit of certain procedures (similar to the quality control of professional practice for physicians) and a complaint-investigation system. Information systems are the cornerstones of quality-assurance programs. These systems are not highly developed in Québec's regions, however, apart from the area covered by Urgences-santé. It should be emphasized that continuing-education initiatives have helped enhance the competencies of the personnel already in place, but these efforts have varied from one region to another.

Recommendation 9: It is recommended that a horizon-scanning system be established to actively monitor emerging evidence in the field of pre-hospital care.

Rationale: Scientific data on all aspects of pre-hospital care should be monitored regularly and continuously so that we can identify procedures that should be part of primary-care or advanced-care services. Structured mechanisms for tracking these data are to be determined. They should include operating procedures ensuring that data are subjected to rigorous and objective analysis. Expert committees on pre-hospital care that are part of recognized bodies such as the Association des médecins d'urgence du Québec (AMUQ) and the Association des spécialistes en médecine d'urgence du Québec (ASMUQ) could be involved in this process.

Download Full Report at: http://www.jephc.com/Vol4_Issue2/AETMISFullReport.pdf 
View Editorial by Dr. Reiner Banken and Dr. Véronique Déry "Introduction of advanced life support to prehospital emergency medical services in Quebec: Can it work? Does it work? Should we do it? How should do we do it?". Journal of Emergency Primary Health Care. 2206;4(2). http://www.jephc.com/full_article.cfm?content_id=365.

\section{Acknowledgement}

The Journal of Emergency Primary Health Care gratefully acknowledges the authors and the Agence d'évaluation des technologies et des modes d'intervention en santé (AETMIS) for granting permission to publish this report. 